\title{
Three-Month Daily Consumption of Sugar-Sweetened Beverages Affects the Liver, Adipose Tissue, and Glucose Metabolism
}

\author{
Ghayoung Lee, Ji Hye Han, Hyo Jin Maeng, Soo Lim* \\ Department of Internal Medicine, Seoul National University Bundang Hospital, Seoul National University College of Medicine, Seongnam, Korea
}

Background: Growing evidence suggests links between sugar-sweetened beverages (SSBs) and metabolic disorders. We investigated the effects of SSBs commonly consumed by adolescents and their relationships to glucose metabolism and fatty liver.

Methods: We treated 7-week old male C57BL/6 mice with water (control) or one of three different SSBs, carbonated soda (Coca-Cola), sweetened milk coffee (Maxwell), or chocolate-added cocoa (Choco-Latte), for 13 weeks ( $n=10$ in each group). Half of the animals were fed a regular chow diet and the other half a high-fat diet ( $40 \%$ fat). Body composition and biochemical variables were investigated at the end of treatment. Histology of the liver and adipose tissue, as well as molecular signaling related to glucose and lipid metabolism, were also evaluated.

Results: During the 13-week treatment, mice treated with chocolate-added cocoa or sweetened milk coffee showed significantly greater increases in body weight compared with controls, especially when fed a high-fat diet. Fasting glucose level was higher in the three SSB-treated groups compared with the control group. Lipid droplets in the liver, fat cell size, and number of CD68-positive cells in adipose tissue were greater in the SSBtreated groups than in the control group. SSB treatments increased the expression of genes related to inflammatory processes in the liver and adipose tissue. Phosphorylation of AKT and glycogen synthase kinase in muscle was significantly reduced in SSB-treated groups.

Conclusion: Daily consumption of SSBs over 3 months lead to metabolic impairment and weight gain and may contribute to development of metabolic diseases.

Key words: Sugar-sweetened beverage, Fatty liver, Glucose homeostasis, Diabetes mellitus, Insulin resistance

\author{
Received July 30, 2019 \\ Reviewed August 26, 2019 \\ Accepted October 4, 2019 \\ ${ }^{*}$ Corresponding author \\ Soo Lim \\ https://orcid.org/0000-0002-4137-1671 \\ Department of Internal Medicine, Seoul \\ National University Bundang Hospital, \\ Seoul National University College of \\ Medicine, 82 Gumi-ro 173beon-gil, \\ Bundang-gu, Seongnam 13620, Korea \\ Tel: +82-31-787-7035 \\ Fax: +82-31-787-4051 \\ E-mail:limsoo@snu.ac.kr
}

\section{INTRODUCTION}

Excessive sugar consumption contributes to risk of cardiometabolic diseases. ${ }^{1}$ Despite global efforts to reduce consumption of sugar, it continues to exceed levels recommended by nutritional guidelines and is an important risk factor for cardiometabolic disease worldwide. ${ }^{1-3}$ Sugar-sweetened beverages (SSBs) are a significant source of added sugars and have been identified as a critical con- tributor to high sugar intake. ${ }^{4}$ In the United States (US), consumption of SSBs is steadily increasing, especially among children and adolescents. ${ }^{5}$ Many Asian countries are following this global nutrition transition toward greater consumption of sugar and SSBs. ${ }^{6-8}$

Overconsumption of SSBs is linked to adverse health outcomes ranging from simple dental problems ${ }^{9}$ to overweight or obesity. ${ }^{10}$ Such changes ultimately lead to increases in metabolic abnormalities including fatty liver, dyslipidemia, type 2 diabetes mellitus

Copyright (C) 2020 Korean Society for the Study of Obesity

(a) This is an Open Access article distributed under the terms of the Creative Commons Attribution Non-Commercial License (https://creativecommons.org/licenses/by-nc/4.o/) which permits unrestricted non-commercial use, distribution, and reproduction in any medium, provided the original work is properly cited. 
(T2DM), and obesity. ${ }^{11} \mathrm{~A}$ recent meta-analysis of seven prospective cohort studies ( $\mathrm{n}=308,420$ participants; age range, 34-75 years) reported $13 \%$ greater risk of stroke and $22 \%$ greater risk of myocardial infarction associated with SSB consumption. ${ }^{12}$

The harmful effects of SSBs are most prominent in the young. Notably, teenagers consume more sugar than other age groups, more than two times the recommended amount. ${ }^{13}$ Students may easily access SSBs such as carbonated soda, energy drinks, sports drinks, and highly sweetened coffees and teas at school. Such unhealthy behavior puts teenagers at risk of obesity and obesity-related diseases. ${ }^{14,15}$ Due to concerns about health-related effects of SSBs, vending machines selling soft drinks have been banned in many parts of the US. In 2014-2015, the U.S. Department of Agriculture nutritional standards for foods and beverages required US high schools participating in federally reimbursable meal programs to remove virtually all SSBs at competitive venues. ${ }^{16}$ Despite these efforts, SSB consumption continues to increase and appears to be an independent risk factor for metabolic diseases. ${ }^{17,18}$

Obesity has emerged as a global public health concern. The prevalence of obesity is continuously increasing due to nutritional transitions in both developing and developed countries ${ }^{19}$ and has increased by $40 \%$ in men and $30 \%$ in women in the US, with greater increases in adolescents. ${ }^{20,21}$ In South Korea, the proportion of people with body mass index $\geq 25 \mathrm{~kg} / \mathrm{m}^{2}$ was $26.0 \%$ in 1998 , which increased to $29.2 \%$ in 2005 and $31.7 \%$ in 2007. Among children and adolescents $<20$ years of age, the prevalence of obesity in Korea doubled over the last decade from 5.8\% in 1997 to $10.9 \%$ in 2007 (Ministry of Health and Welfare: Healthy Eating Styles for Korean Adults; https://www.mw.go.kr/front_new/al/sal0301vw.jsp?PAR MENU_ID $=04 \&$ MENU_ID $=0403 \& C O N T \_S E Q=223072 \&$ page $=1$ ). Furthermore, the prevalence of metabolic syndrome in youths aged 12 to 19 years in South Korea increased from $4.0 \%$ in 1998 to $7.6 \%$ in 2007, along with major increases in dyslipidemia and abdominal obesity. ${ }^{22}$ Overweight and obese adolescents in the US obtain $>300 \mathrm{kcal}$ per day from SSBs, an average of $15 \%$ of their total daily energy intake. ${ }^{23}$ Thus, SSB consumption is likely to be a primary contributor to obesity and metabolic diseases in the young.

Despite the great concern about health risks related to SSB consumption, the unfavorable metabolic effects of daily consumption of SSBs have not been clearly demonstrated, especially in younger people. Here, we aimed to investigate the effects of SSBs on glucose metabolism and fatty liver disease using an animal model.

\section{METHODS}

\section{Selection of SSBs}

Korean national data show that adolescents and young adults in South Korea frequently consume SSBs, including carbonated sodas, energy drinks, sports drinks, and highly sweetened coffees and teas. ${ }^{24}$ To identify a representative sample, we conducted a survey of SSB consumption among Korean high school students $(\mathrm{n}=101)$. The most popular beverages consumed were chocolate-added cocoa (Choco-Latte; Lotte Chilsung, Seoul, Korea), carbonated soda (Coca-Cola or Sprite; The Coca-Cola Company, Atlanta, GA, USA), and sweetened milk coffee (Maxwell, Dongsuh, Seoul, Korea; Let's Be, Lotte Chilsung; Santa Fe, Paldo Food, Seoul, Korea). We selected a representative brand from each category, Choco-Latte, CocaCola, and Maxwell, to investigate their impacts on development of fatty liver and glucose metabolism. The volume of one portion, total calories, sugar content, and proportion of sugar contained in each beverage are shown in Table 1. Each drink contains between 11 and $18 \mathrm{~g}$ of sugar per $100 \mathrm{~g}$.

\section{Experimental design and animal care}

Forty male C57BL/6 mice aged 7 weeks (Japan SLC, Shizuoka, Japan) were used in the study. All mice were maintained at a temperature of $22^{\circ} \mathrm{C} \pm 2^{\circ} \mathrm{C}$ and $55 \% \pm 10 \%$ humidity under a $12: 12$ hour light-dark cycle and were allowed food and water ad libitum for the duration of the treatment period.

After being matched for weight, study mice were divided into two groups: one group was fed a normal chow diet and the second a high-fat diet. Each group was further divided into four subgroups with various treatments: water, equivalent of two cans of Coca-Cola, equivalent of two cans of Maxwell, and equivalent of two packs of Choco-Latte. Therefore, there were eight groups total, with five mice per group. At 8 AM each day, treatment beverages were administered to study animals using a feeding device. The amount of beverage administered to mice was calculated based on body weight according to a commonly used conversion between humans $(60 \mathrm{~kg})$ and mice $(20 \mathrm{~g})$. At the end of the 13-week treatment, the mice 
Table 1. Beverages used in the study and their nutritional components

\begin{tabular}{|c|c|c|c|}
\hline Variable & $\begin{array}{l}\text { Carbonated } \\
\text { soda } \\
\text { (Coca-Cola) }\end{array}$ & $\begin{array}{c}\text { Sweetened milk } \\
\text { coffee } \\
\text { (Maxwell) }\end{array}$ & $\begin{array}{c}\text { Chocolate-added } \\
\text { cocoa } \\
\text { (Choco-Latte) }\end{array}$ \\
\hline Volume (mL)/can or pack & 250 & 175 & 235 \\
\hline Total calories (kcal)/can or pack & 112 & 65 & 160 \\
\hline \multicolumn{4}{|l|}{ Sugar } \\
\hline Amount $(\mathrm{g}) /$ can or pack & 27 & 13 & 27 \\
\hline Amount/volume (g/mL) & 0.108 & 0.074 & 0.115 \\
\hline \multicolumn{4}{|l|}{ Protein } \\
\hline Amount $(\mathrm{g}) /$ can or pack & 0 & 1 & 3.5 \\
\hline Amount/volume (g/mL) & - & 0.006 & 0.015 \\
\hline \multicolumn{4}{|l|}{ Fat } \\
\hline Amount $(\mathrm{g}) /$ can or pack & 0 & 1.4 & 2.5 \\
\hline Amount/volume (g/mL) & - & 0.008 & 0.011 \\
\hline \multicolumn{4}{|l|}{ Saturated fat } \\
\hline Amount (g)/can or pack & 0 & 0.7 & 2.5 \\
\hline Amount/volume (g/mL) & - & 0.004 & 0.011 \\
\hline \multicolumn{4}{|l|}{ Cholesterol } \\
\hline Amount $(\mathrm{mg}) /$ can or pack & 0 & 5 & 8 \\
\hline Amount/volume (mg/mL) & - & 0.029 & 0.034 \\
\hline Sodium (mg) & 15 & 85 & 150 \\
\hline \multicolumn{4}{|c|}{ Daily amount administered to mice equivalent to 2 cans or packs for humans* } \\
\hline Administered volume $(\mathrm{mL})^{\dagger}$ & 0.17 & 0.12 & 0.16 \\
\hline Administered sugar (mg) & 18.0 & 8.7 & 18.0 \\
\hline Administered protein (mg) & 0 & 0.7 & 2.3 \\
\hline Administered fat (mg) & 0 & 0.9 & 1.7 \\
\hline Administered saturated fat (mg) & 0 & 0.5 & 1.7 \\
\hline Administered cholesterol (mg) & 0 & 0.0033 & 0.0053 \\
\hline
\end{tabular}

*Volume was calculated using translation from human $(60 \mathrm{~kg})$ to 5-week-old mouse $(20 \mathrm{~g}){ }^{\dagger}{ }^{\dagger}$ Rounded to three decimal points.

were anesthetized with an intraperitoneal injection of a mixture of zolazepam/tiletamine ( $80 \mathrm{mg} / \mathrm{kg}$, Zoletil 50; Virbac, Carros, France) and xylazine $(20 \mathrm{mg} / \mathrm{kg}$, Rompun; Bayer HealthCare, Leverkusen, Germany) for euthanasia.

This study was approved by the Institutional Animal Care and Use Committee of Seoul National University Bundang Hospital (No. 51-2015-031). Experiments were performed in compliance with the Guide for Experimental Animal Research of the Laboratory for Experimental Animal Research, Clinical Research Institute, Seoul National University Bundang Hospital, Korea.

\section{Body weight, body composition, and food intake measurements}

During the 13-week treatment, body weight was measured once per week. Food and water intakes of all mice in each study group were also measured every week throughout the treatment period. Body composition was determined by dual-energy X-ray absorptiometry using a PIXImus II apparatus (GE Lunar Corp., Madison, WI, USA) at the end of the treatment period.

\section{Biochemical markers associated with cardiovascular risk}

Fasting blood glucose concentration was measured every third week using a glucometer (Accu-Chek Inform II Blood Glucose Meter; Roche Diagnostics, Mannheim, Germany). The mice were subjected to an intraperitoneal glucose tolerance test after 13 weeks of treatment. Each animal was injected intraperitoneally with $1.5 \mathrm{~g} / \mathrm{kg}$ of a $50 \mathrm{~mol} / \mathrm{L}$ glucose solution. Blood samples (about $10 \mu \mathrm{L}$ ) were collected from an incision in the tail at $0,15,30,60,90$, and 120 minutes after the glucose load. The area under the curve for glucose (AUC $\left.C_{\text {glucose }}\right)$ was calculated from 0 to 120 minutes using the trapezoid rule for glucose data.

An insulin tolerance test (ITT) was also conducted at the end of the treatment period. The mice were intraperitoneally injected with insulin ( $1 \mathrm{U} / \mathrm{kg}$; Humalog, Eli Lilly, Indianapolis, IN, USA) after 4 to 5 hours of fasting. Blood glucose level was measured at 0,15 , $30,60,90$, and 120 minutes after insulin administration.

Blood samples were collected from mice after 8 hours of fasting at the end of the treatment period. Plasma glucose concentration was measured using a glucose oxidase method (YSI 2300-STAT; YSI, Yellow Springs, OH, USA) immediately after the blood was drawn. Insulin level was measured at baseline and at the end of the treatment period using a mouse-specific radioimmunoassay kit (Millipore, Billerica, MA, USA). Lipid profiles (triglycerides, highdensity lipoprotein-cholesterol, and low-density lipoprotein-cholesterol), liver enzymes (aspartate [AST] and alanine aminotransferase $[\mathrm{ALT}])$, and serum creatinine concentrations were determined using enzymatic procedures (Hitachi 747 Chemistry Analyzer; Hitachi, Tokyo, Japan). The levels of tumor necrosis factor- $\alpha$ (TNF- $\alpha$ ), interleukin-6 (IL-6), and monocyte chemoattractant protein-1 (MCP-1) were measured using a Multiplex Assay Kit (RADPK-81K; Millipore). Adiponectin and high-sensitivity C-reactive protein (hsCRP) concentrations were measured using enzyme-linked immunosorbent assay kits developed by Millipore and BD Biosciences Pharmingen (Heidelberg, Germany), respectively. To estimate pancreatic $\beta$-cell function and insulin resistance, the 
homeostasis assessment of insulin resistance (HOMA-IR) and homeostasis assessment of $\beta$-cell function (HOMA- $\beta$ ) were calculated using fasting insulin and glucose levels. ${ }^{25}$

\section{Histology of liver and adipose tissues}

After 13 weeks of treatment, liver and visceral adipose tissues were harvested to investigate the effects of SSBs on histology. Tissue samples were fixed in formalin, routinely processed, and embedded in paraffin. Paraffin-embedded sections $(4 \mu \mathrm{m})$ were stained with hematoxylin and eosin $(\mathrm{H} \& \mathrm{E})$. The area of lipid droplets that had accumulated in the liver was measured using a microscope for image acquisition and corresponding software for quantification (Image J software; National Institutes of Health, Bethesda, MD, USA). ${ }^{26}$ The sizes of fat cells in abdominal visceral fat were measured using the same software. Crown-like structures, which indicate clustering of inflammatory cells, were identified.

\section{Immunofluorescence staining of CD68}

Deparaffinized and rehydrated sections were steamed in target retrieval solution ( $\mathrm{pH}$ 9.0; Dako, Carpinteria, CA, USA) for antigen retrieval and washed in phosphate-buffered saline. The sections were blocked with 5\% normal goat serum (Gibco-BRL, Rockville, MD, USA) for 2 hours at room temperature (RT), incubated with primary antibody overnight at $4^{\circ} \mathrm{C}$, and then incubated with secondary antibody for 1 hour at RT. Anti-CD68 antibody (Abcam, Cambridge, MA, USA) was used as the primary antibody. Alexa 488-conjugated goat anti-rabbit IgG (Invitrogen, Grand Island, NY, USA) was used as the secondary antibody. After mounting in Vectashield mounting medium containing DAPI (4',6-diamidino-2-phenylindole; Vector Laboratories, Burlingame, CA, USA), images were acquired using a fluorescence microscope (IX81; Olympus, Tokyo, Japan).

\section{Western blot analysis}

Tissues were lysed in lysis buffer, and protein concentration was determined using a protein assay kit (Pierce Biotechnology, Rockford, IL, USA). Proteins (30 $\mu \mathrm{g})$ were resolved on 10\% SDS-PAGE (sodium dodecyl sulfate polyacrylamide gel electrophoresis) and transferred onto a PVDF membrane (Millipore). Nonspecificbinding sites on the membrane were blocked in tris buffered saline with Tween (TBS-T) 20 containing 5\% bovine serum albumin for 2 hours at RT and incubated with the primary antibody overnight at $4^{\circ} \mathrm{C}$. After three washes with TBS-T, the membrane was blocked and incubated with the secondary antibody for 1 hour at RT. The following primary antibodies were used: anti-Akt, anti-phosphoAkt, anti-glycogen synthase kinase-3 $\beta$ (GSK3 $\beta$ ), and antiphospho-GSK3 $\beta$ antibodies (Cell Signaling Laboratories, Beverly, MA, USA). Horseradish peroxidase-conjugated anti-rabbit IgG (Santa Cruz Biotechnology, Santa Cruz, CA, USA) was used as the secondary antibody.

\section{Quantitative real-time polymerase chain reaction for} genes related to metabolism, inflammation, and endoplasmic reticulum stress

Total RNA was extracted from tissue samples using an Isol-RNA Lysis Reagent (5 Prime, Gaithersburg, MD, USA) according to the manufacturer's recommendations. From $1 \mu \mathrm{g}$ of total RNA, complementary DNA (cDNA) was synthesized using an iScript cDNA Synthesis Kit (Bio-Rad, Hercules, CA, USA). The expression of adiponectin, IL-6, C/EBP homologous protein (CHOP), and MCP-1 in the abdominal visceral adipose tissue and the expression of CHOP, glucose-regulated protein-78 (GRP78), and sterol regulatory element binding transcription factor-1c (SREBP1c) in the liver were analyzed using a Power SYBR Green PCR Master Mix (Applied Biosystems, Warrington, UK) on an ABI 7500 system (Applied Biosystems). We used 18S rRNA as an internal control.

\section{Statistical analysis}

Results are reported as mean \pm standard deviation or standard error of the mean where indicated. Analysis of variance with posthoc analysis was used to determine significant mean differences among groups. A commercial statistical package was used for statistical analysis IBM SPSS version 20.0 (IBM Corp., Armonk, NY, USA). A $P<0.05$ was considered significant in all tests of statistical inference.

\section{RESULTS}

\section{Effect of SSBs on body weight}

For 13 weeks, starting from 7 weeks of age, weight changes were 

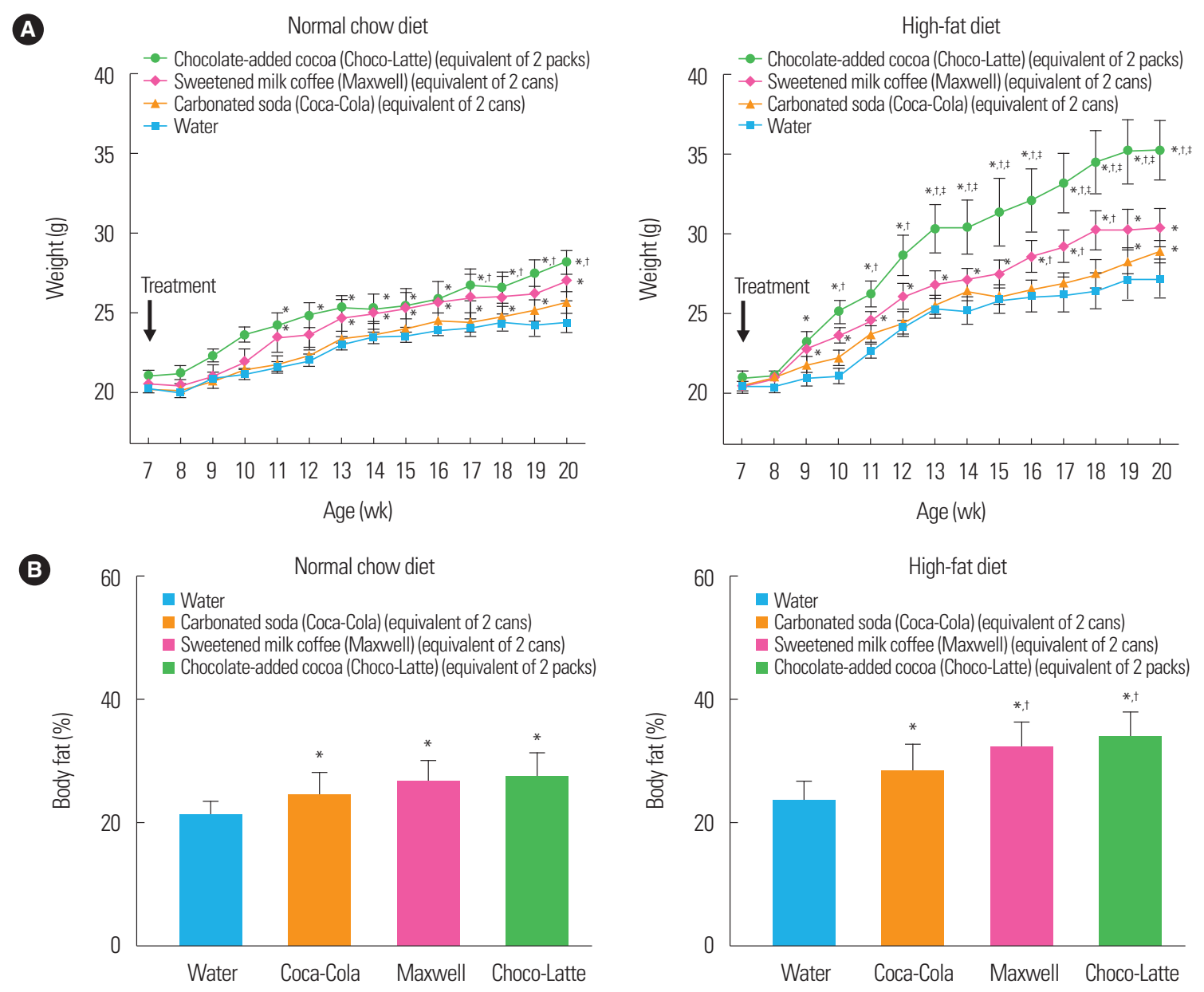

C
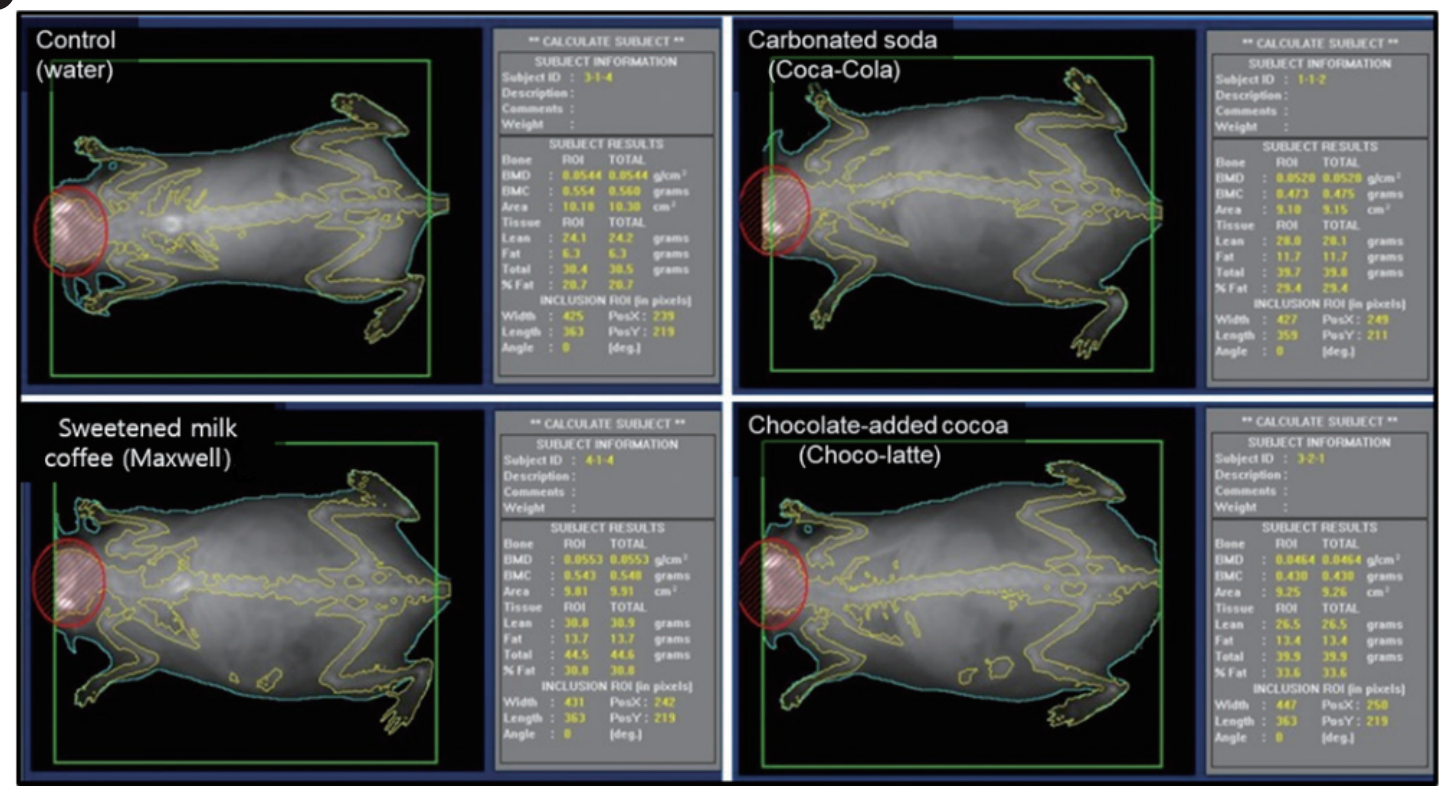

Figure 1. Changes in weight during treatment $(\mathrm{A})$ and body fat percentage at the end of the treatment $(\mathrm{B})$ in mice treated with water, chocolate-added cocoa, sweetened milk coffee, and carbonated soda on a normal chow diet or high-fat diet ( ${ }^{*}$ vs. water; ${ }^{\dagger}$ vs. carbonated soda; ${ }^{\ddagger}$ vs. sweetened milk coffee, all $\left.P<0.05\right)$. (C) Representative photos of dual-energy $X$-ray absorptiometry for body fat measurement. 
compared between control, carbonated soda, sweetened milk coffee, and chocolate-added cocoa treatment groups (Fig. 1). The mice fed a high-fat diet significantly increased in weight during the treatment period compared with those allowed a normal chow diet. The SSB-treated groups treated with chocolate-added cocoa or sweetened milk coffee showed greater increases in body weight compared to the group treated with water, and the differences were more prominent in those allowed a high-fat diet. At the end of the treatment period, body weight of the chocolate-added cocoa group had increased to a significantly greater extent compared with the other three groups allowed a high-fat diet (Fig. 1A).

Body fat percentages were higher in the three groups treated with SSBs than in the control group, and the differences were more prominent in those allowed a high-fat diet (Fig. 1B). In those allowed a high-fat diet, body fat percentages in the groups treated with chocolate-added cocoa or sweetened milk coffee increased more than in the group treated with carbonated soda.

\section{Effects of SSBs on blood glucose, glycemic excursion,} and insulin tolerance

We analyzed the effects of SSBs on glucose homeostasis. At the end of the treatment period, fasting blood glucose level in all SSB groups was significantly increased compared with the control group (Supplementary Fig. 1A). Of the three SSB-treated groups fed a normal chow diet, blood glucose level during the glucose tolerance test and ITT did not differ significantly from that of the control group (Supplementary Fig. 1B, C). Under the high-fat diet condition, blood glucose level during the glucose tolerance test increased more at 15, 30, and 60 minutes in all three SSB-treated groups than it did in the control group (Supplementary Fig. 1B). The $\mathrm{AUC}_{\text {glucose }}$ was also higher in the three SSB-treated groups than in the control group. Glucose level in the ITT was higher in the chocolate-added cocoa and sweetened milk coffee groups than in the control group, particularly at 0,15, 30, and 60 minutes (Supplementary Fig. 1C). Those in the carbonated soda group had higher glucose level than the control group at 60 minutes.

\section{Effects of SSBs on overall food and water intake}

We determined cumulative food and water intakes in the four treatment groups. There were no significant differences in food or water consumption between the mice fed a normal chow diet, except at 16 and 17 weeks of treatment (Supplementary Fig. 2). In

Table 2. Weight and biochemical measurement including glucose homeostasis, inflammatory markers, and adiponectin levels in treatment groups of mice fed a normal chow diet after 13 weeks

\begin{tabular}{|c|c|c|c|c|}
\hline Variable & Control (water) & Carbonated soda (Coca-Cola) & Sweetened milk coffee (Maxwell) & Chocolate-added cocoa (Choco-Latte) \\
\hline Weight (g) & $23.3 \pm 3.6$ & $25.6 \pm 3.6^{*}$ & $27.0 \pm 3.2^{*,+}$ & $28.2 \pm 3.6^{*,+}$ \\
\hline Fasting glucose (mmol/L) & $4.9 \pm 0.8$ & $5.1 \pm 1.1^{*}$ & $5.3 \pm 1.2^{*}$ & $6.1 \pm 1.3^{*}$ \\
\hline Fasting insulin ( $\mu \mid \mathrm{U} / / \mathrm{L})$ & $9.1 \pm 1.2$ & $11.3 \pm 1.3$ & $11.5 \pm 1.3^{*}$ & $12.1 \pm 1.4^{*}$ \\
\hline HOMA-IR ${ }^{\S}$ & $1.98 \pm 0.21$ & $2.56 \pm 0.36^{*}$ & $2.70 \pm 0.45^{*}$ & $3.28 \pm 0.51^{*}$ \\
\hline HOMA- $\beta$ & $131.4 \pm 23.1$ & $140.6 \pm 28.3$ & $129.7 \pm 34.1$ & $92.9 \pm 23.1^{*}$ \\
\hline AST (U/L) & $47.6 \pm 8.8$ & $57.2 \pm 6.9^{*}$ & $61.5 \pm 9.0^{*}$ & $63.1 \pm 7.7^{*}$ \\
\hline ALT (U/L) & $34.6 \pm 7.7$ & $44.4 \pm 10.3^{*}$ & $57.0 \pm 7.2^{*, t}$ & $55.0 \pm 9.1^{*, t}$ \\
\hline Triglycerides (mmol/L) & $1.4 \pm 0.3$ & $1.6 \pm 0.5^{*}$ & $1.6 \pm 0.6^{*, \dagger}$ & $1.7 \pm 0.7^{*}$ \\
\hline HDL cholesterol (mmol/L) & $2.1 \pm 0.3$ & $2.0 \pm 0.5$ & $1.8 \pm 0.5$ & $1.8 \pm 0.5$ \\
\hline LDL cholesterol (mmol/L) & $5.6 \pm 0.6$ & $5.7 \pm 0.7$ & $5.8 \pm 0.7$ & $5.9 \pm 0.8$ \\
\hline hsCRP (mg/L) & $0.22 \pm 0.25$ & $0.56 \pm 0.26^{*}$ & $0.44 \pm 0.14^{*}$ & $0.68 \pm 0.28^{*}$ \\
\hline Adiponectin $(\mu \mathrm{g} / \mathrm{mL})$ & $9.3 \pm 2.4$ & $7.6 \pm 2.1^{*}$ & $7.3 \pm 2.6^{*}$ & $6.7 \pm 2.1^{*}$ \\
\hline TNF- $\alpha(p g / m L)$ & $8.1 \pm 3.5$ & $12.2 \pm 6.0$ & $10.3 \pm 3.0^{*}$ & $11.7 \pm 4.4^{*}$ \\
\hline IL-6 (pg/mL) & $4.8 \pm 1.1$ & $5.8 \pm 1.9$ & $5.2 \pm 1.2$ & $5.0 \pm 1.8$ \\
\hline MCP-1 (ng/mL) & $3.5 \pm 1.7$ & $4.8 \pm 1.4^{*}$ & $4.7 \pm 2.1^{*}$ & $5.6 \pm 1.5^{*}$ \\
\hline
\end{tabular}

Values are presented as mean \pm standard deviation. Post-hoc analysis by least significant difference t-tests for mean differences between two groups.

${ }^{*}$ vs. control; ${ }^{\dagger}$ vs. carbonated soda; $P<0.05$ in all cases; ${ }^{\S} H O M A-I R=(\mu \mathrm{U} / \mathrm{L} \times \mathrm{mmol} / \mathrm{L})$.

HOMA-IR, homeostasis assessment of insulin resistance; HOMA- $\beta$, homeostasis assessment of $\beta$-cell function; AST, aspartate aminotransferase; ALT, alanine aminotransferase; HDL, high-density lipoprotein; LDL, low-density lipoprotein; hsCRP, high-sensitivity C-reactive protein; TNF- $\alpha$, tumor necrosis factor- $\alpha$; IL-6, interleukin-6; MCP-1, monocyte chemoattractant protein-1. 
Table 3. Weight and biochemical measures including glucose homeostasis, inflammatory markers, and adiponectin levels in treatment groups of mice fed a high-fat diet after 13 weeks

\begin{tabular}{|c|c|c|c|c|}
\hline Variable & Control (water) & Carbonated soda (Coca-Cola) & Sweetened milk coffee (Maxwell) & Chocolate-added cocoa (Choco-Latte) \\
\hline Weight (g) & $27.2 \pm 3.3$ & $28.8 \pm 3.7^{*}$ & $30.3 \pm 3.2^{*}$ & $35.3 \pm 5.6^{*,+, \pm}$ \\
\hline Fasting glucose (mmol/L) & $6.7 \pm 0.9$ & $7.7 \pm 1.2^{*}$ & $7.6 \pm 1.1^{*}$ & $8.6 \pm 1.1^{*}$ \\
\hline Fasting insulin ( $\mu \mid \mathrm{U} / / \mathrm{L})$ & $11.2 \pm 1.0$ & $13.5 \pm 1.2^{*}$ & $14.7 \pm 1.3^{*}$ & $16.1 \pm 1.4^{*}$ \\
\hline HOMA-IR ${ }^{\S}$ & $3.34 \pm 0.82$ & $4.63 \pm 0.73^{*}$ & $4.97 \pm 0.81^{*}$ & $6.16 \pm 0.93^{*,+}$ \\
\hline HOMA- $\beta$ & $69.6 \pm 13.3$ & $64.0 \pm 24.0$ & $71.6 \pm 19.2$ & $63.1 \pm 28.6$ \\
\hline AST (U/L) & $49.2 \pm 9.8$ & $62.3 \pm 8.2^{*}$ & $68.6 \pm 6.6^{*}$ & $72.1 \pm 8.1^{*}$ \\
\hline $\mathrm{ALT}(\mathrm{U} / \mathrm{L})$ & $46.6 \pm 9.7$ & $51.4 \pm 11.3^{*}$ & $59.3 \pm 7.8^{*, \dagger}$ & $65.2 \pm 5.8^{*, t}$ \\
\hline Triglycerides (mmol/L) & $1.9 \pm 1.3$ & $2.2 \pm 0.8^{*}$ & $2.3 \pm 0.7^{*}$ & $2.4 \pm 0.7^{*}$ \\
\hline HDL cholesterol (mmol/L) & $1.9 \pm 0.6$ & $1.7 \pm 0.5$ & $1.7 \pm 0.6$ & $1.6 \pm 0.4^{*}$ \\
\hline LDL cholesterol (mmol/L) & $5.9 \pm 0.8$ & $5.9 \pm 0.7$ & $6.1 \pm 0.8$ & $6.2 \pm 1.1^{*}$ \\
\hline hsCRP (mg/L) & $0.61 \pm 0.21$ & $0.78 \pm 0.16^{*}$ & $0.89 \pm 0.21^{*}$ & $0.72 \pm 0.18^{*}$ \\
\hline Adiponectin ( $\mu \mathrm{g} / \mathrm{mL})$ & $8.3 \pm 2.1$ & $6.6 \pm 2.0^{*}$ & $6.3 \pm 2.1^{*}$ & $5.8 \pm 1.7^{*, \dagger}$ \\
\hline TNF- $\alpha(p g / m L)$ & $11.1 \pm 3.6$ & $13.2 \pm 3.6^{*}$ & $12.3 \pm 4.0^{*, t}$ & $15.7 \pm 3.4^{*, t}$ \\
\hline IL-6 (pg/mL) & $5.8 \pm 1.2$ & $5.5 \pm 1.9$ & $5.8 \pm 1.6$ & $6.1 \pm 1.8$ \\
\hline MCP-1 (ng/mL) & $5.5 \pm 1.4$ & $5.9 \pm 1.8^{*}$ & $6.7 \pm 1.5^{*}$ & $7.6 \pm 1.5^{*, \dagger}$ \\
\hline
\end{tabular}

Values are presented as mean \pm standard deviation. Post-hoc analysis by least significant difference t-tests for mean differences between two groups.

${ }^{*}$ vs. control; ${ }^{\top}$ vs. carbonated soda; ${ }^{\dagger}$ vs. sweetened milk coffee; $P<0.05$ in all cases; ${ }^{5} \mathrm{HOMA}-\mathrm{IR}=(\mu \mathrm{lU} / \mathrm{L} \times \mathrm{mmol} / \mathrm{L})$.

HOMA-IR, homeostasis assessment of insulin resistance; HOMA- $\beta$, homeostasis assessment of $\beta$-cell function; AST, aspartate aminotransferase; ALT, alanine aminotransferase; HDL, high-density lipoprotein; LDL, low-density lipoprotein; hsCRP, high-sensitivity C-reactive protein; TNF- $\alpha$, tumor necrosis factor- $\alpha$; IL-6, interleukin-6; MCP-1, monocyte chemoattractant protein-1.

contrast, there were significant increases in food and water consumption in the chocolate-added cocoa group and sweetened milk coffee group from 4 to 5 weeks of treatment compared with the control group or carbonated soda-fed groups allowed access to a high-fat diet. Thus, the milk-containing beverage groups (sweetened milk coffee and chocolate-added cocoa) showed a trend toward higher cumulative caloric intake of food and drinks compared with the control and carbonated soda groups, particularly when allowed a high-fat diet.

\section{Effects of SSBs on biochemical parameters}

When fed a normal chow diet, fasting glucose and insulin levels were higher in SSB-treated groups than controls, resulting in high HOMA-IR and low HOMA- $\beta$. Liver enzyme activities of AST and ALT and circulating levels of triglycerides, hsCRP, and MCP-1 were also higher in these groups than they were in control mice (Table 2).

When fed a high-fat diet, the differences in glucose, insulin, AST, ALT, triglycerides, and MCP-1 levels between the SSB-treated groups and control mice were more prominent. HOMA-IR increased substantially in mice in the SSB-treated groups allowed a high-fat diet compared to mice fed a normal chow diet. Adiponec- tin level was lower and TNF- $\alpha$ level was higher in the SSB-treated groups than in controls (Table 3 ).

\section{Effects of SSB supplementation on liver and adipose tissue histology}

Liver sections from mice either on a normal chow diet or a highfat diet were stained by $\mathrm{H} \& \mathrm{E}$ at the end of the treatment (Fig. 2A). After 13 weeks of treatment, the area of lipid droplets in the livers of mice in the SSB-treated groups was larger than in control mice allowed a high-fat diet (Fig. 2B).

After 13 weeks of treatment, the fat cells in abdominal visceral adipose tissue were larger in the three SSB-treated groups than in control mice (Fig. 3). Crown-like structures were found in mice in the three SSB-treated groups but not in the control group. These differences were only observed in mice fed a high-fat diet and not in those fed a normal chow diet (data not shown).

\section{Immunofluorescence staining of CD68 in adipose tissue}

Supplementary Fig. 3 shows immunofluorescence staining results for inflammatory cells. The number of CD68-positive cells increased in mice in the three SSB-treated groups compared with 

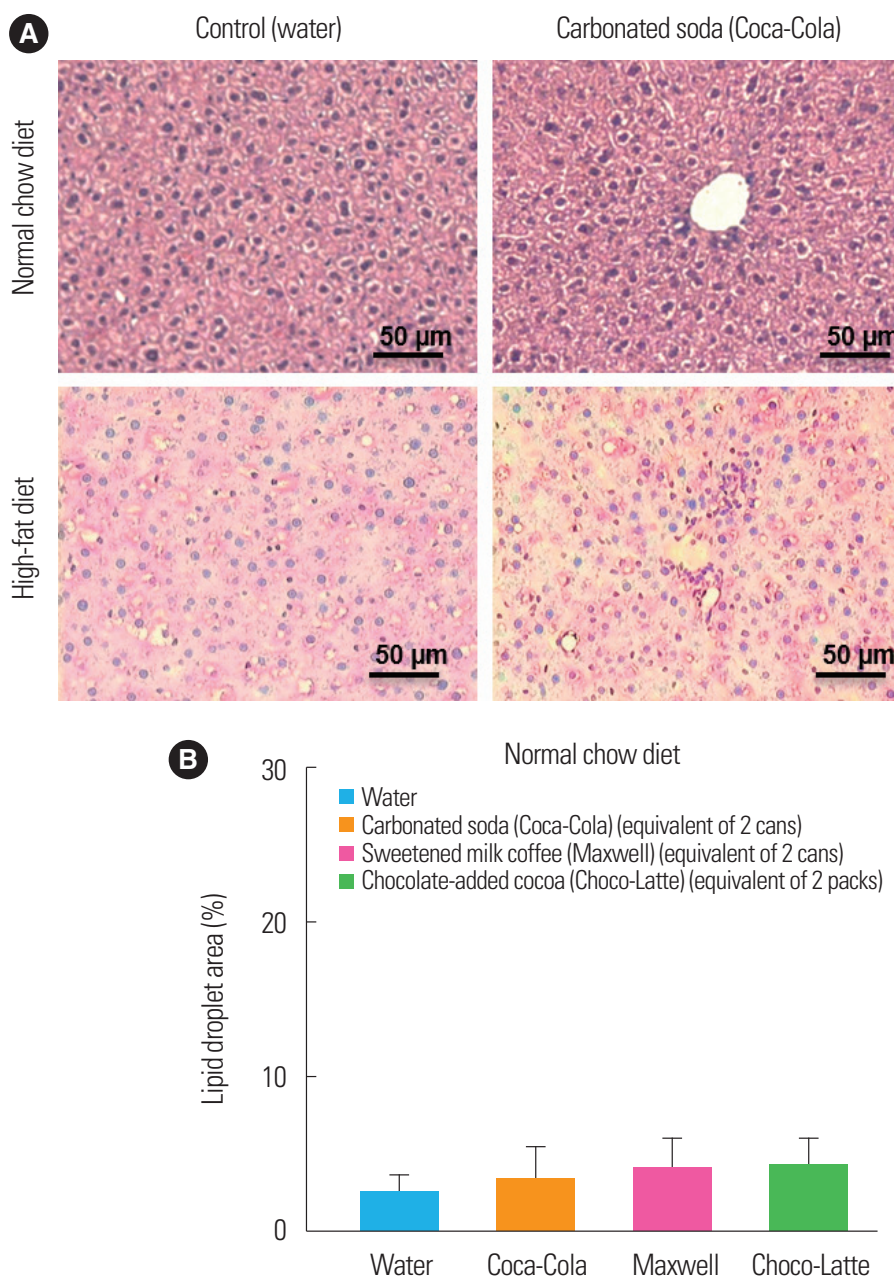

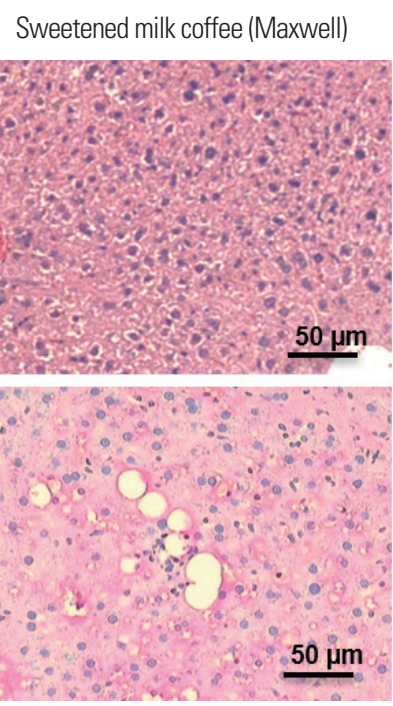

Chocolate-added cocoa (Choco-Latte)
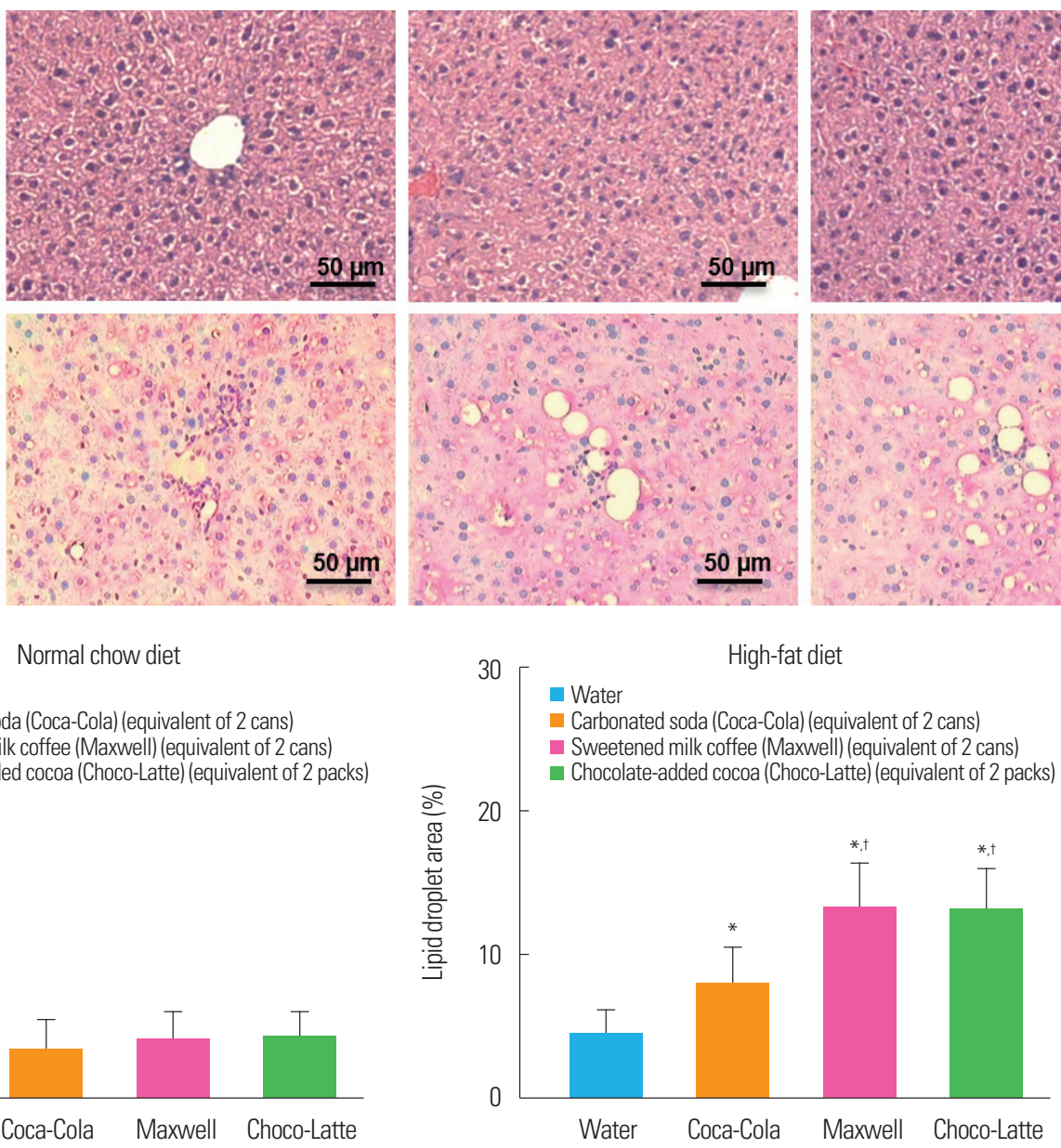

Figure 2. (A) Liver histology by H\&E staining. All images were acquired at $\times 200$ ( $n=5$ per group). (B) The percentage area of lipid droplets in the liver (\% of total area). Values are presented as mean \pm standard error of mean. ${ }^{*} P<0.05$ vs. water; ${ }^{\dagger} P<0.05$ vs. carbonated soda.

mice treated with water.

\section{Relative expression of pAKT and pGSK in muscle of mice allowed a high-fat diet}

We investigated the relative expression of pAKT and pGSK in the muscle tissues of the four treatment groups (Supplementary Fig. 4). The pAKT to AKT ratio was markedly lower in the SSBtreated groups than in the control group. Moreover, the levels in mice treated with chocolate-added cocoa or sweetened milk coffee were further attenuated compared with those in mice treated with carbonated soda (Supplementary Fig. 4B). The pGSK to GSK ratio was also significantly lower in the SSB-treated groups than it was in the control group. Significant differences were observed only in mice fed a high-fat diet and not in those fed a normal chow diet (data not shown).

\section{Effects of SSB treatment on expression of genes related} to metabolism, inflammation, endoplasmic reticulum stress, and lipid metabolism in adipose tissue and liver

The level of expression of adiponectin in visceral adipose tissue was significantly lower and the levels of IL-6 and CHOP were significantly higher in the three SSB-treated groups compared with the control group for mice fed a high-fat diet (Supplementary Fig. 5). There were no significant differences in levels between the SSBtreated groups. However, MCP-1 expression in visceral adipose tissue was increased only in mice treated with carbonated soda or sweetened milk coffee compared with controls.

In the liver, the levels of expression of CHOP and GRP78 
A

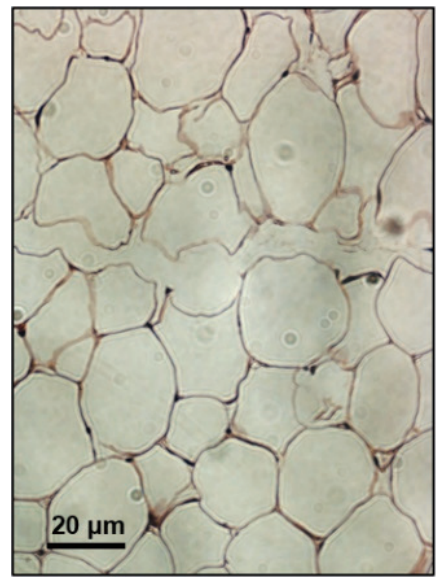

Control (water)

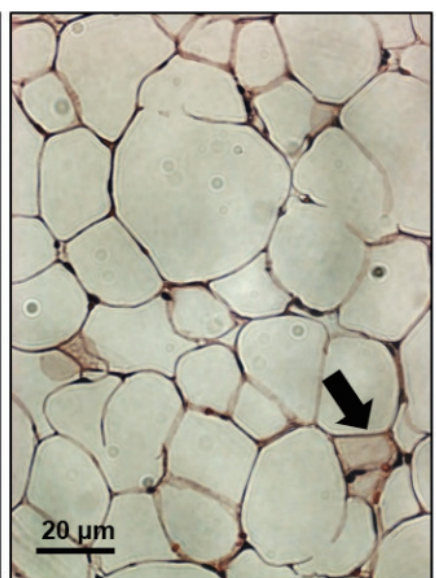

Carbonated soda (Coca-Cola)

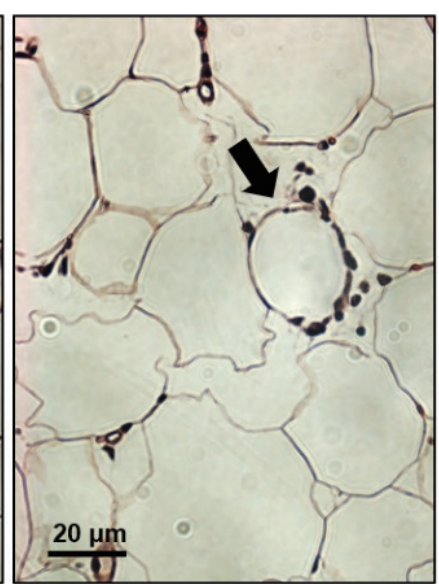

Sweetened milk coffee (Maxwell)

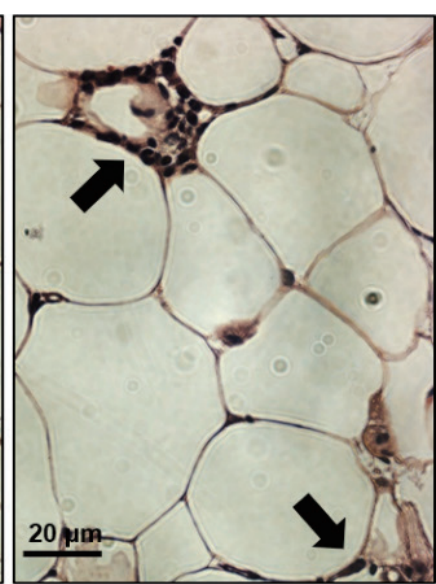

Chocolate-added cocoa (Choco-Latte)
B

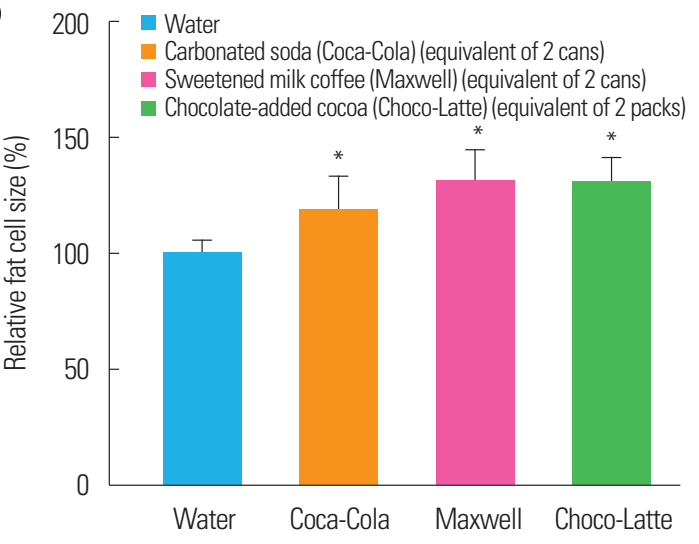

mRNA were increased in the three SSB-treated groups, but MCP1 expression was higher only in the group treated with sweetened milk coffee (Supplementary Fig. 6). The expression of CHOP in the group treated with chocolate-added cocoa was greater than that in groups treated with carbonated soda or sweetened milk coffee. The expression of SREBP1c mRNA was significantly attenuated in groups treated with SSBs compared with the control groups.

\section{DISCUSSION}

In the present study, treatment with SSBs for 13 weeks resulted in greater increase in body weight and blood glucose concentration than treatment with water. Mice in groups treated with SSB had greater amount of body fat and greater infiltration of inflammatory cells and deposition of fat in their livers and adipose tissue.

Based on a survey conducted for the present study, we selected a single representative of each category of beverage: Coca-Cola for
Figure 3. (A) Histology of abdominal visceral adipose tissue stained with H\&E in high-fat diet groups ( $n=5$ per group). The arrows indicate crown-like structures. (B) Comparison of fat cell size ( $\%$ of control). Values are presented as mean \pm standard error of mean. ${ }^{*} P<0.05$ vs. control (water).

carbonated sodas, Maxwell for sweetened milk coffees, and ChocoLatte for chocolate-added cocoas. After 13 weeks of treatment, in the groups of mice treated with chocolate-added cocoa and sweetened milk coffee, the increase in body weight was greater than in controls consuming either diet. The fasting blood glucose level was significantly higher in mice in the SSB-treated groups than in the control mice in both diet groups. Notably, the increases in body weight and fasting glucose level were more prominent in SSB-treated mice allowed access to a high fat diet. Fasting glucose concentration in the SSB-treated mice exceeded $7 \mathrm{mmol} / \mathrm{L}(126 \mathrm{mg} / \mathrm{dL})$, which would be classified as diabetes in humans. This finding indicates that the high-fat diet exacerbated metabolic deterioration induced by SSB treatment.

A recent study has shown that high intake of sugars from SSBs, such as sugar-added tea and coffee, is associated with hyperglycemia and inflammatory markers. ${ }^{18}$ Metabolically, the harmful effects of SSBs are mostly the contribution of fructose and additional calo- 
ries. In the liver, fructose easily converts to hepatic glycogen or fat, promotes lipogenesis, and worsens insulin resistance. ${ }^{27,28}$ Therefore, fructose consumption is appreciated as a risk factor for obesity or cardiometabolic diseases. ${ }^{29}$ Consistent with such concerns, adverse metabolic effects of SSBs were clearly observed in the present study, which showed increased fat accumulation in the liver and attenuated expression of genes related to insulin sensitivity in SSBtreated mice. Moreover, SSBs provide a source of de novo lipogenesis and inhibit adipose lipolysis, ${ }^{30,31}$ ultimately increasing visceral adiposity. ${ }^{32}$ The present study also found that adipocytes in mice treated with SSBs were enlarged and had more crown-like structures. These changes may also contribute to peripheral insulin resistance and inflammation.

Muscle is a primary tissue that responds to insulin after a glucose load. The SSB-treated mice showed impaired insulin signaling in muscle, as determined by AKT and GSK phosphorylation, compared to control mice. A fructose-rich diet has detrimental effects on mitochondrial function and energy metabolism in muscle. ${ }^{33,34}$ In agreement with previous findings, glucose metabolism in muscle was impaired in the mice treated with SSBs, which may contribute to development of diabetes.

Glucose and lipid metabolism are interrelated and are affected by cytokines (e.g., adiponectin, TNF- $\alpha$, and IL-6) and molecules involved in inflammation, oxidative stress, and endoplasmic reticulum (ER) stress (e.g., hsCRP, MCP-1, and plasminogen activator inhibitor-1). In the present study, aggravated insulin resistance in SSB-treated mice was accompanied by increased levels of expression of $\mathrm{CHOP}$, GRP78, and ER stress markers in the liver. ${ }^{35}$ Thus, SSB treatment is likely to promote inflammatory- and stress-related processes in metabolically active organs.

In the present study, we observed metabolically harmful effects of SSBs in mice fed a high-fat diet, while mice fed a normal chow diet were less affected. The biochemical and histological changes in the SSB-treated groups occurred mainly through increased fat accumulation and altered lipid metabolism in metabolically active organs. Therefore, such changes might be more exacerbated in mice fed a high-fat diet, which already have a vulnerable signaling pathway related to lipid metabolism as a result of the fat load.

The World Health Organization has advised reduction of sugar intake to avoid chronic diseases such as fatty liver disease, T2DM, and metabolic disorders. ${ }^{36,37}$ Despite great concern about the metabolic risks of added sugar, consumption of SSBs is steadily increasing, especially among younger people. ${ }^{4}$ In addition, people tend to consume diverse types of SSBs together, for example, carbonated sodas and sweetened milk coffee. This may intensify the harmful effects of these SSBs through de novo lipogenesis and inhibit adipose lipolysis, ${ }^{30,31}$ resulting in fat accumulation in the abdomen and metabolically active organs, such as liver and muscle. ${ }^{32}$ The combined consumption of SSBs may also aggravate inflammation and insulin resistance by altering expression of related genes, such as IL-6 and MCP- $1 .{ }^{38}$

Given that SSBs are consumed mainly by adolescents and young adults, and their negative effects emerge as quickly as 3 months, it would be prudent to advise teenagers to consume less SSBs. This would be one of the most effective strategies to avoid further increases in metabolic diseases such as fatty liver, dyslipidemia, and diabetes mellitus.

The strengths of the present study are as follows. First, application of in-depth evaluation of body composition and histological assessments of liver and fat tissue produced a comprehensive analysis of the metabolic effects of SSBs. Second, the SSBs used in this study were chosen based on a survey conducted in a high school in Korea and thus may reflect actual consumption. Finally, experiments were performed using groups of mice fed a normal diet or a high-fat diet, which demonstrated differences in effects of SSBs based on type of diet.

Nonetheless, one possible limitation of this research is that a 13week treatment period might have been insufficient. Conducting the experiment for a longer period would have made it possible to observe the effects of chronic exposure to SSBs.

In conclusion, the equivalent of two cans or packs of SSBs consumed daily for 13 weeks led to harmful biochemical and histological effects on energy homeostasis. These effects included increased body weight and body fat along with infiltration of inflammatory cells, which induced fat accumulation in the liver and ultimately impaired insulin sensitivity. Expression of genes related to energy metabolism, inflammation, and ER stress was affected unfavorably by SSB treatment. These changes may confer a greater risk of future metabolic disease in exposed persons. Thus, concerns regarding the harmful effects of SSB consumption are warranted. 


\section{CONFLICTS OF INTEREST}

The authors declare no conflict of interest.

\section{ACKNOWLEDGMENTS}

This research was funded by Servier Korea (Seoul, Korea) through a subcontract with Seoul National University Bundang Hospital, (Seongnam, Korea; No. 06-2015-129). The funding agency had no role in the study design, data collection and analysis, decision to publish, or preparation of the manuscript.

\section{AUTHOR CONTRIBUTIONS}

Study concept and design: SL; acquisition of data: GL, JHH, HJM, and SL; analysis and interpretation of data: SL; drafting of the manuscript: HJM and SL; critical revision of the manuscript: GL, HJM, and SL; statistical analysis: SL; obtained funding: SL; administrative, technical, or material support: all authors; and study supervision: SL.

\section{SUPPLEMENTARY MATERIALS}

Supplementary Figures 1-6 can be found via https://doi.org/10. 7570/jomes19042.

\section{REFERENCES}

1. Yang Q, Zhang Z, Gregg EW, Flanders WD, Merritt R, Hu FB. Added sugar intake and cardiovascular diseases mortality among US adults. JAMA Intern Med 2014;174:516-24.

2. Popkin BM, Nielsen SJ. The sweetening of the world's diet. Obes Res 2003;11:1325-32.

3. Austin GL, Ogden LG, Hill JO. Trends in carbohydrate, fat, and protein intakes and association with energy intake in normal-weight, overweight, and obese individuals: 1971-2006. Am J Clin Nutr 2011;93:836-43.

4. Bremer AA, Byrd RS, Auinger P. Racial trends in sugar-sweetened beverage consumption among US adolescents: 1988-2004. Int J Adolesc Med Health 2011;23:279-86.
5. Johnson RK, Appel LJ, Brands M, Howard BV, Lefevre M, Lustig $\mathrm{RH}$, et al. Dietary sugars intake and cardiovascular health: a scientific statement from the American Heart Association. Circulation 2009; 120:1011-20.

6. Kwak JH, Jo G, Chung HK, Shin MJ. Association between sugar-sweetened beverage consumption and incident hypertension in Korean adults: a prospective study. Eur J Nutr 2019; 58:1009-17.

7. Gui ZH, Zhu YN, Cai L, Sun FH, Ma YH, Jing J, et al. Sugarsweetened beverage consumption and risks of obesity and hypertension in Chinese children and adolescents: a national cross-sectional analysis. Nutrients 2017;9:E1302.

8. He B, Long W, Li X, Yang W, Chen Y, Zhu Y. Sugar-sweetened beverages consumption positively associated with the risks of obesity and hypertriglyceridemia among children aged 7-18 years in South China. J Atheroscler Thromb 2018;25:81-9.

9. Park S, Pan L, Sherry B, Li R. The association of sugar-sweetened beverage intake during infancy with sugar-sweetened beverage intake at 6 years of age. Pediatrics 2014;134 Suppl 1: S56-62.

10. Malik VS, Pan A, Willett WC, Hu FB. Sugar-sweetened beverages and weight gain in children and adults: a systematic review and meta-analysis. Am J Clin Nutr 2013;98:1084-102.

11. Reedy J, Krebs-Smith SM. Dietary sources of energy, solid fats, and added sugars among children and adolescents in the United States. J Am Diet Assoc 2010;110:1477-84.

12. Narain A, Kwok CS, Mamas MA. Soft drinks and sweetened beverages and the risk of cardiovascular disease and mortality: a systematic review and meta-analysis. Int J Clin Pract 2016;70: 791-805.

13. Lee HS, Kwon SO, Yon M, Kim D, Lee JY, Nam J, et al. Dietary total sugar intake of Koreans: based on the Korea National Health and Nutrition Examination Survey (KNHANES), 20082011. J Nutr Health 2014;47:268-76.

14. Centers for Disease Control and Prevention (CDC). Beverage consumption among high school students: United States, 2010. MMWR Morb Mortal Wkly Rep 2011;60:778-80.

15. Kondaki K, Grammatikaki E, Jiménez-Pavón D, De Henauw S, González-Gross M, Sjöstrom M, et al. Daily sugar-sweetened beverage consumption and insulin resistance in European ado- 
lescents: the HELENA (Healthy Lifestyle in Europe by Nutrition in Adolescence) Study. Public Health Nutr 2013;16: 479-86.

16. Food and Nutrition Service, USDA. National School Lunch Program and School Breakfast Program: nutrition standards for all foods sold in school as required by the Healthy, HungerFree Kids Act of 2010: interim final rule. Fed Regist 2013;78: 39067-120.

17. Hu FB, Malik VS. Sugar-sweetened beverages and risk of obesity and type 2 diabetes: epidemiologic evidence. Physiol Behav 2010;100:47-54.

18. O’Connor L, Imamura F, Brage S, Griffin SJ, Wareham NJ, Forouhi NG. Intakes and sources of dietary sugars and their association with metabolic and inflammatory markers. Clin Nutr 2018;37:1313-22.

19. Popkin BM, Adair LS, Ng SW. Global nutrition transition and the pandemic of obesity in developing countries. Nutr Rev 2012;70:3-21.

20. Yang L, Colditz GA. Prevalence of overweight and obesity in the United States, 2007-2012. JAMA Intern Med 2015;175: 1412-3.

21. Rodríguez LA, Madsen KA, Cotterman C, Lustig RH. Added sugar intake and metabolic syndrome in US adolescents: crosssectional analysis of the National Health and Nutrition Examination Survey 2005-2012. Public Health Nutr 2016;19: 2424-34.

22. Lim S, Jang HC, Park KS, Cho SI, Lee MG, Joung H, et al. Changes in metabolic syndrome in American and Korean youth, 1997-2008. Pediatrics 2013;131:e214-22.

23. Wang YC, Bleich SN, Gortmaker SL. Increasing caloric contribution from sugar-sweetened beverages and 100\% fruit juices among US children and adolescents, 1988-2004. Pediatrics 2008;121:e1604-14.

24. Han E, Kim TH, Powell LM. Beverage consumption and individual-level associations in South Korea. BMC Public Health 2013;13:195.

25. Matthews DR, Hosker JP, Rudenski AS, Naylor BA, Treacher DF, Turner RC. Homeostasis model assessment: insulin resistance and beta-cell function from fasting plasma glucose and insulin concentrations in man. Diabetologia 1985;28:412-9.
26. Stringer DM, Zahradka P, Declercq VC, Ryz NR, Diakiw R, Burr LL, et al. Modulation of lipid droplet size and lipid droplet proteins by trans-10, cis-12 conjugated linoleic acid parallels improvements in hepatic steatosis in obese, insulin-resistant rats. Biochim Biophys Acta 2010;1801:1375-85.

27. Samuel VT. Fructose induced lipogenesis: from sugar to fat to insulin resistance. Trends Endocrinol Metab 2011;22:60-5.

28. Schwarz JM, Noworolski SM, Wen MJ, Dyachenko A, Prior JL, Weinberg ME, et al. Effect of a high-fructose weight-maintaining diet on lipogenesis and liver fat. J Clin Endocrinol Metab 2015;100:2434-42.

29. Malik VS, Hu FB. Fructose and cardiometabolic health: what the evidence from sugar-sweetened beverages tells us. J Am Coll Cardiol 2015;66:1615-24.

30. Rizkalla SW, Luo J, Guilhem I, Boillot J, Bruzzo F, Chevalier A, et al. Comparative effects of 6 week fructose, dextrose and starch feeding on fat-cell lipolysis in normal rats: effects of isoproterenol, theophylline and insulin. Mol Cell Biochem 1992;109: 127-32.

31.Luo J, Rizkalla SW, Lerer-Metzger M, Boillot J, Ardeleanu A, Bruzzo F, et al. A fructose-rich diet decreases insulin-stimulated glucose incorporation into lipids but not glucose transport in adipocytes of normal and diabetic rats. J Nutr 1995;125: 164-71.

32. Stanhope KL, Schwarz JM, Keim NL, Griffen SC, Bremer AA, Graham JL, et al. Consuming fructose-sweetened, not glucosesweetened, beverages increases visceral adiposity and lipids and decreases insulin sensitivity in overweight/obese humans. J Clin Invest 2009;119:1322-34.

33. Crescenzo R, Bianco F, Coppola P, Mazzoli A, Cigliano L, Liverini G, et al. The effect of high-fat--high-fructose diet on skeletal muscle mitochondrial energetics in adult rats. Eur J Nutr 2015;54:183-92.

34. Crescenzo R, Bianco F, Coppola P, Mazzoli A, Cigliano L, Liverini $G$, et al. Increased skeletal muscle mitochondrial efficiency in rats with fructose-induced alteration in glucose tolerance. Br J Nutr 2013;110:1996-2003.

35. Kim I, Xu W, Reed JC. Cell death and endoplasmic reticulum stress: disease relevance and therapeutic opportunities. Nat Rev Drug Discov 2008;7:1013-30. 
36. Sakurai M, Nakamura K, Miura K, Takamura T, Yoshita K, Nagasawa SY, et al. Sugar-sweetened beverage and diet soda consumption and the 7-year risk for type 2 diabetes mellitus in middle-aged Japanese men. Eur J Nutr 2014;53:251-8.

37. Ma J, Fox CS, Jacques PF, Speliotes EK, Hoffmann U, Smith $\mathrm{CE}$, et al. Sugar-sweetened beverage, diet soda, and fatty liver disease in the Framingham Heart Study cohorts. J Hepatol 2015;63:462-9.

38. Kanda H, Tateya S, Tamori Y, Kotani K, Hiasa K, Kitazawa R, et al. MCP-1 contributes to macrophage infiltration into adipose tissue, insulin resistance, and hepatic steatosis in obesity. J Clin Invest 2006;116:1494-505. 